\title{
On the Critical Behaviour of Dyson's Quantum Hierarchical Models
}

\author{
Carlos H.C. Moreira, Ricardo S. Schor \\ Departamento de Física, Instituto de Ciências Exatas, Universidade Federal de Minas Gerais, \\ C.P. 702, CEP 30161-970 Belo Horizonte, Brazil
}

Received: 26 March 1993

\begin{abstract}
We study the probability distribution of the appropriately scaled square of the total spin for critical asymptotically hierarchical quantum models and show that it converges, as the number of spins tends to infinity, to the same function related to the corresponding classical systems. Thus, we exhibit explicitly a property of statistical mechanical systems which, at the critical point, does not depend whether one uses a classical or quantum mechanical description.
\end{abstract}

\section{Introduction}

It is widely believed that the critical behaviour of statistical mechanical system is independent of the way (classical or quantum mechanical) used to describe it. Sewell [8] has shown quite generally that the long distance behaviour of critical quantum systems is classical, but the question on the relation between this classical behaviour and the one corresponding to the classical version of the system analyzed was not considered.

In the last decade, rigorous renormalization group methods have been developed, which permitted, among other things, a deep understanding of some classical critical systems, particularly those having infrared asymptotically free behaviour [6]. On the other hand, no such progress has been achieved to treat quantum critical systems in general - a task much more difficult than the classical case because of the appearance of non-commutative objects.

In this paper we consider a special class of spin systems which can be analyzed both classically and quantum mechanically: the asymptotically hierarchical models in the terminology of [9]. These are generalizations of Dyson's hierarchical models, originally introduced in [4]. The classical version of these models, with the spins taking values \pm 1 , at the critical point was first studied in a rigorous way in the fundamental paper by Bleher and Sinai [3]. Vector valued classical spins at low temperatures were analyzed by Bleher and Major in $[1,2]$ and by Schor and O'Carroll in [7], using a different hierarchical model. Here we consider instead quantum mechanical spin $\frac{1}{2}$ systems and compare them with the corresponding classical three dimensional vector models. Specifically, we study the probability 
distribution of the appropriately scaled square of the total spin for critical quantum systems and show that it converges as the number of spins tends to infinity to the same function related to the corresponding classical systems. Thus we exhibit explicitly a property of statistical mechanical systems which at the critical point does not depend whether one uses a classical or quantum mechanical description.

We now explain the organization of the paper. In Sect. 2 we define the class of hierarchical quantum and classical spin systems which will be analyzed and show, in heuristic terms, why the probability distributions mentioned above should be the same in both cases at the critical point. In Sect. 3 we state our main theorem, the proof of which is given in Sect. 4 and follows basically the ideas developed in [9], which presents a simplified treatment of the original proof for the scalar hierarchical models [3]. As a corollary we also obtain a multiscale expansion for the free energy associated to the systems of Sect. 2.

\section{Definition of the Models and Main Results}

To each site of the lattice $\Lambda_{n}=\left\{1,2, \ldots, 2^{n}\right\}$ we associate a spin variable which may be a classical scalar variable: $S_{i}= \pm 1$, a classical vector: $\vec{S}_{i} \in \mathbf{R}^{3}$, or a quantum $\operatorname{spin} \frac{1}{2} \cdot \vec{S}_{i}=I \otimes \ldots \otimes \frac{1}{2} \vec{\sigma} \otimes \ldots \otimes I$ acting on $\left(\mathbb{C}^{2}\right)^{2^{n}}$, where the components of $\vec{\sigma}$ are the usual Pauli matrices and $\frac{1}{2} \vec{\sigma}$ is placed in the $i^{\text {th }}$ position in the definition of $\vec{S}_{i}$.

A member of the class of asymptotically hierarchical models is specified by an initial Hamiltonian $H_{n_{0}}\left(\vec{S}_{1}, \ldots, \vec{S}_{2^{n_{0}}}\right)$ defined on $\Lambda_{n_{0}}$. The Hamiltonians $H_{n}\left(\vec{S}_{1}, \ldots, \vec{S}_{2^{n}}\right)$ on $\Lambda_{n}$ for $n>n_{0}$ are given recursively by

$$
\begin{aligned}
H_{n}\left(\vec{S}_{1}, \ldots, \vec{S}_{2^{n}}\right)= & H_{n-1}\left(\vec{S}_{1}, \ldots, \vec{S}_{2^{n-1}}\right)+H_{n-1}\left(\vec{S}_{2^{n-1}+1}, \ldots, \vec{S}_{2^{n}}\right) \\
& -\left(\frac{c}{4}\right)^{n}\left(\sum_{i \in \Lambda_{n}} \vec{S}_{i}\right)^{2} .
\end{aligned}
$$

We will assume that $H_{n_{0}}$ is rotationally invariant. As shown in Dyson's paper [4], the thermodynamic limit exists if $c<2$ and there is phase transition if $c>1$. We assume in the sequel $1<c<2$.

Consider the characteristic function of $\left(\sum_{i \in \Lambda_{n}} \vec{S}_{i}\right)^{2}$ in the canonical ensemble defined by $H_{n_{0}}$. Classically,

$$
\begin{aligned}
C_{n}^{(c)}(\xi) & =\left\langle\exp \left[i \xi\left(\sum_{i \in \Lambda_{n}} \vec{S}_{i}\right)^{2}\right]\right\rangle_{n} \\
& =\frac{1}{Z_{n}^{(c)}} \int \exp \left[i \xi\left(\sum_{i \in \Lambda_{n}} \vec{S}_{i}\right)^{2}-\beta H_{n}\right] d^{3} S_{1} \ldots d^{3} S_{2^{n}}
\end{aligned}
$$

where $\xi \in \mathbf{R}, Z_{n}^{(c)}$ is the classical partition function and $\beta$ is the inverse temperature. If $p_{n}^{(c)}(s)$ is the distribution of $\sum_{i \in \Lambda_{n}} \vec{S}_{i}$ (which depends only on $\left|\sum_{i \in \Lambda_{n}} \vec{S}_{i}\right|$ ),

$$
C_{n}^{(c)}(\xi)=\int_{0}^{\infty} \exp \left(i \xi s^{2}\right) p_{n}^{(c)}(s) 4 \pi s^{2} d s
$$


It follows easily from $(2.1)$ that the function $p_{n}^{(c)}(s)$ satisfies the recursion

$$
p_{n}^{(c)}(s)=\frac{Z_{n-1}^{(c)^{2}}}{Z_{n}^{(c)}} \exp \left[\beta\left(\frac{c}{4}\right)^{n} s^{2}\right] \int p_{n-1}^{(c)}(|\vec{u}|) p_{n-1}^{(c)}\left(\left|s \hat{e}_{1}-\vec{u}\right|\right) d^{3} u
$$

where $\hat{e}_{1}$ is the unit vector in the one-direction.

Proceeding heuristically as in [9], we expect that above the critical temperature $|\vec{s}|^{2} \sim 2^{n}$ as $n \rightarrow \infty$ and hence the exponential in (2.4) should be irrelevant in this case. On the other hand, below the critical temperature $|\vec{s}|^{2} \sim 2^{2 n}$ and the exponential is dominant. At the critical temperature we may expect $|\vec{s}|^{2} \sim 2^{2 n}$ and the exponential is dominant. At the critical temperature we may expect $|\vec{s}|^{2} \sim\left(\frac{4}{c}\right)^{n}$ and if this is indeed true, the characteristic function of $\left(\frac{c}{4}\right)^{n}\left(\sum_{i \in \Lambda_{n}} \vec{S}_{i}\right)^{2}$ should converge to

$$
C^{(c)}(\xi)=\lim _{n \rightarrow \infty} C_{n}^{(c)}\left(\left(\frac{c}{4}\right)^{n} \xi\right)=\int_{0}^{\infty} \exp \left(i \xi u^{2}\right) q^{(c)}(u) 4 \pi u^{2} d u
$$

with the distribution $q^{(c)}(u)$ satisfying the relation

$$
q^{(c)}(u)=\text { const } \exp \left(\beta u^{2}\right) \int q^{(c)}(|\vec{v}|) q^{(c)}\left(\left|\frac{2}{\sqrt{c}} u \vec{e}_{1}-\vec{v}\right|\right) d^{3} v .
$$

The constant above is determined by the condition $C^{(c)}(0)=1$.

The formal considerations were shown to be true for the scalar $S_{i}= \pm 1$ models by Bleher and Sinai [3], who studied the problem under the restriction $\sqrt{2}<c<2$. In this case, the transformation on the space of probability distributions defined by the right-hand side of (2.6) has a Gaussian fixed point which is thermodynamically stable, i.e. the linearized map has only one eigenvalue greater than one.

The arguments for the scalar models can be extended to the vector models so as to justify the picture above. The Gaussian solution of $(2.6)$ is

$$
q^{(c)}(u)=\left(\frac{\alpha(\beta)}{\pi}\right)^{\frac{3}{2}} \exp \left[-\alpha(\beta) u^{2}\right]
$$

where $\alpha(\beta)=\beta c /(2-c)$.

We consider now the quantum case. Let $\Lambda_{p}^{(j)}=\left\{(j-1) 2^{p}+1\right.$, $\left.(j-1) 2^{p}+2, \ldots,(j-1) 2^{p}+2^{p}\right\}$ for $1 \leqq p \leqq n$ and $1 \leqq j \leqq 2^{n-p}$. Define

$$
\vec{\mu}_{p}^{(j)}=\sum_{i \in \Lambda_{p}^{(j)}} \vec{S}_{i}
$$

Then, as pointed out in Dyson's original paper [4], the set $\left\{\left(\vec{\mu}_{p}^{(j)}\right)^{2}: 1 \leqq p \leqq n\right.$, $\left.1 \leqq j \leqq 2^{n-p} ;\left(\vec{\mu}_{n}^{(1)}\right)_{3}\right\}$ (where $\left(\vec{\mu}_{n}^{(1)}\right)_{3}$ is the third component of $\left.\vec{\mu}_{n}^{(1)}\right)$ is maximally commuting in $\left(\mathbb{C}^{2}\right)^{2^{n}}$ and the common eigenfunctions diagonalize $H_{n}$. Denoting the eigenfunctions by $\left|\left\{\ell_{p}^{(j)}\right\} ; m_{n}\right\rangle$, we have

$$
\begin{aligned}
& \left(\vec{\mu}_{r}^{(k)}\right)^{2}\left|\left\{\ell_{p}^{(j)}\right\} ; m_{n}\right\rangle=\ell_{r}^{(k)}\left(\ell_{r}^{(k)}+1\right)\left|\left\{\ell_{p}^{(j)}\right\} ; m_{n}\right\rangle, \\
& \left(\vec{\mu}_{n}^{(1)}\right)_{3}\left|\left\{\ell_{p}^{(j)}\right\} ; m_{n}\right\rangle=m_{n}\left|\left\{\ell_{p}^{(j)}\right\} ; m_{n}\right\rangle .
\end{aligned}
$$


Since $\vec{\mu}_{p}^{(j)}=\vec{\mu}_{p-1}^{(2 j-1)}+\vec{\mu}_{p-1}^{(2 j)}(1 \leqq p \leqq n)$, we must have $\left|\ell_{p-1}^{(2 j-1)}-\ell_{p-1}^{(2 j)}\right| \leqq \ell_{p}^{(j)}$ $\leqq \ell_{p-1}^{(2 j-1)}+\ell_{p-1}^{(2 j)}$ and initially $\ell_{1}^{(j)} \in\{0,1\}$. Also, the possible values of $m_{n}$ are restricted to $\left|m_{n}\right| \leqq \ell_{n}^{(1)}$.

In terms of the basis (2.9) the characteristic function of $\left(\sum_{i \in A_{n}} \vec{S}_{i}\right)^{2}$ in the quantum case is

$$
C_{n}^{(a)}(\xi)=\frac{1}{Z_{n}^{(q)}} \sum_{\left\{\ell_{p}^{(j)}\right\}}^{\prime}\left(2 \ell_{n}^{(1)}+1\right) \exp \left[i \xi \ell_{n}^{(1)}\left(\ell_{n}^{(1)}+1\right)-\beta H_{n}\left(\left\{\ell_{r}^{(k)}\right\}\right)\right],
$$

where $Z_{n}^{(q)}$ is the quntum partition function and the prime in the summation takes into account the restriction imposed on the set of possible values for $\left\{\ell_{p}^{(j)}\right\}$, alluded to above.

Writing (2.10) as

$$
C_{n}^{(q)}(\xi)=\frac{1}{Z_{n}^{(q)}} \sum_{\ell=0}^{2^{n-1}}(2 \ell+1) \exp [i \xi \ell(\ell+1)] z_{n}(\ell),
$$

it is easy to verify, using (2.1), that

$$
z_{n}(\ell)=\exp \left[\beta\left(\frac{c}{4}\right)^{n} \ell(\ell+1)\right] \sum_{\substack{\ell_{i} \in\left\{0|| \ell_{1}-\ell_{2} \mid \leqq \ell \leqq 2^{n-2}\right\}}} z_{n-1}\left(\ell_{1}\right) z_{n-1}\left(\ell_{2}\right) .
$$

Proceeding in analogy with the classical case we consider the characteristic functions of $\left(\frac{c}{4}\right)^{n}\left(\sum_{i \in A_{n}} \vec{S}_{i}\right)^{2}$, which can be written as

$$
\begin{aligned}
C_{n}^{(q)}\left(\left(\frac{c}{4}\right)^{n} \xi\right)= & \frac{2}{Z_{n}^{(q)}}\left(\frac{2}{\sqrt{c}}\right)^{n} \sum_{u \in \mathrm{A}_{n}}\left[u+\frac{1}{2}\left(\frac{\sqrt{c}}{2}\right)^{n}\right] \\
& \times \exp \left\{i \xi u\left[u+\left(\frac{\sqrt{c}}{2}\right)^{n}\right]\right\} z_{n}\left(\left(\frac{2}{\sqrt{c}}\right)^{n} u\right),
\end{aligned}
$$

where $\mathbf{A}_{n}=\left\{\left(\frac{\sqrt{c}}{2}\right)^{n} \ell: 0 \leqq \ell \leqq 2^{n-1}\right\}$. If the analogy can indeed be carried over to the quantum case, we may expect that at critical $\beta$ the limit of $C_{n}^{(q)}\left(\left(\frac{c}{4}\right)^{n} \xi\right)$ exists as $n \rightarrow \infty$ and is given by

$$
C^{(q)}(\xi)=\int_{0}^{\infty} u \exp \left(i \xi u^{2}\right) p^{(q)}(u) d u
$$

with $p^{(q)}(u)=\lim _{n \rightarrow \infty} p_{n}^{(q)}(u), p_{n}^{(q)}(u)=\frac{2}{Z_{n}^{(q)}}\left(\frac{4}{c}\right)^{n} z_{n}\left(\left(\frac{2}{\sqrt{c}}\right)^{n} u\right)$. In addition, since from $(2.12) p_{n}^{(q)}(u)$ satisfies the recursion

$$
p_{n}^{(q)}(u)=\frac{2}{c} \frac{Z_{n-1}^{(q)^{2}}}{Z_{n}^{(q)}} \exp \left\{\beta u\left[u+\left(\frac{\sqrt{c}}{2}\right)^{n}\right]\right\}\left(\frac{c}{4}\right)^{n-1} \sum_{\substack{v_{1}, v_{2} \in A_{n-1} \\\left|v_{1}-v_{2}\right| \leqq \frac{2}{\sqrt{c}} u \leqq v_{1}+v_{2}}} p_{n-1}^{(q)}\left(v_{1}\right) p_{n-1}^{(q)}\left(v_{2}\right),
$$


we may also expect that $p^{(q)}(u)$ satisfies the equation

$$
p^{(q)}(u)=\text { const } \exp \left(\beta u^{2}\right) \quad \iint_{\left|v_{1}-v_{2}\right| \leqq \frac{2}{\sqrt{c}} u \leqq v_{1}+v_{2}} p^{(q)}\left(v_{1}\right) p^{(q)}\left(v_{2}\right) d v_{1} d v_{2} .
$$

Assuming that all steps above can be carried out, we may ask about the relation between the classical and quantum characteristic functions $C^{(c)}(\xi)$ and $C^{(q)}(\xi)$. If we set $p^{(q)}(u)=4 \pi u q^{(q)}(u)$, such that

$$
C^{(q)}(\xi)=\int_{0}^{\infty} \exp \left(i \xi u^{2}\right)\left(q^{(q)}(u) 4 \pi u^{2} d u\right.
$$

then $q^{(q)}(u)$ satisfies the equation

$$
q^{(q)}(u)=\text { const } \exp \left(\beta u^{2}\right) \quad \iint_{\left|v_{1}-v_{2}\right| \leqq \frac{2}{\sqrt{c}} u \leqq v_{1}+v_{2}} q^{(q)}\left(v_{1}\right) q^{(q)}\left(v_{2}\right) \frac{v_{1} v_{2}}{u} d v_{1} d v_{2} .
$$

Now we make the change of variables $\left(v_{1}, v_{2}\right) \rightarrow(r, \theta)$, where $v_{1}=r$, $v_{2}=\sqrt{\frac{4}{c} u^{2}+r^{2}-\frac{4}{\sqrt{c}} u r \cos \theta}$, to get

$$
q^{(q)}(u)=\text { const } \exp \left(\beta u^{2}\right) \int_{0}^{\infty} r^{2} q^{(q)}(r) d r \int_{0}^{\pi} q^{(q)}\left(\sqrt{\frac{4}{c} u^{2}+r^{2}-\frac{4}{\sqrt{c}} u r \cos \theta}\right) \sin \theta d \theta
$$

that is

$$
q^{(q)}(u)=\text { const } \exp \left(\beta u^{2}\right) \int q^{(q)}(|\vec{r}|) q^{(q)}\left(\left|\frac{2}{\sqrt{c}} u \hat{e}_{1}-\vec{r}\right|\right) d^{3} r,
$$

which is the same as (2.6). Thus, provided the heuristic arguments for the quantum case can be justified, we see that the distribution of $\lim _{n \rightarrow \infty}\left(\frac{c}{4}\right)^{n}\left(\sum_{i \in \Lambda_{n}} \vec{S}_{i}\right)^{2}$ at the critical temperature is the same whether one uses classical or quantum Statistical Mechanics.

In this paper we show that the above reasoning holds (for the quantum case) if the parameter $c$ is restricted to $\sqrt{2}<c<2^{4 / 5}$. The region $2^{\frac{4}{5}} \leqq c<2$, which is allowed in the classical case, could not be handled within our method of proof.

The strategy we follow is to study the recursion (2.15) using the methods of the scalar $S_{i}= \pm 1$ case [9]. Unfortunately, we have not been able to implement the very powerful analyticity techniques of Gawedzki and Kupiainen [5], due to the discreteness of the spin values. In our problem we have to deal separately with large fields and also with very small fields, so that the perturbative region corresponds to intermediate values of the fields. The precise meaning of these statements, as well as the formulation of our main theorem, is given in the next section.

\section{Main Theorem}

Definition 1. Given $\beta>0$ and $1<c<2$ define the operator $\mathscr{A}(\beta)$ acting on the probability space $L_{2}\left([0, \infty), \frac{4}{\sqrt{\pi}} \gamma(\beta)^{\frac{3}{2}} x^{2} \exp \left(-\gamma(\beta) x^{2}\right) d x\right), \quad \gamma(\beta)=$ 
$2(c-1) \beta /(2-c)$, henceforth denominated $L_{2}(\beta)$, by

$$
(\mathscr{A}(\beta) f)(x ; \beta)=\left(\frac{8 \alpha(\beta)}{\pi c}\right)^{\frac{3}{2}} \int \exp \left(-\frac{8 \alpha(\beta)}{c}|\vec{r}|^{2}\right) f\left(\frac{2}{\sqrt{c}}\left|\vec{r}+\frac{x}{2} \vec{e}_{1}\right|\right) d^{3} r .
$$

The following properties may be easily verified:

P1. $\mathscr{A}(\beta)$ is a self-adjoint operator on $L_{2}(\beta)$.

P2. $\mathscr{A}(\beta)$ may be explicitly diagonalized: $\mathscr{A}(\beta) \varphi_{n}=c^{-n} \varphi_{n}, \quad n \in \mathbf{N}$, with $\varphi_{n}(x ; \beta)=\left[2^{n+1} \sqrt{(2 n+1) ! \gamma(\beta)} x\right]^{-1} H_{2 n+1}(\sqrt{\gamma(\beta)} x)$, where $H_{2 n+1}$ is the $(2 n+1)^{\text {th }}$ Hermite polynomial $\left(H_{m}(x)=(-1)^{m} \exp \left(x^{2}\right)(\mathrm{d} / \mathrm{d} x)^{m} \exp \left(-x^{2}\right)\right)$. The set $\left\{\varphi_{n}\right\}_{n=0}^{\infty}$ is an orthonormal basis for $L_{2}(\beta)$ and the first polynomials are $\varphi_{0}(x ; \beta)=1, \varphi_{1}(x ; \beta)=\sqrt{\frac{2}{3}} \gamma(\beta) x^{2}-\sqrt{\frac{3}{2}}, \varphi_{2}(x ; \beta)=\sqrt{\frac{2}{15}} \gamma(\beta)^{2} x^{4}-$ $\sqrt{\frac{10}{3}} \gamma(\beta) x^{2}+\sqrt{\frac{15}{8}}$

P3. Regarding $\mathscr{A}(\beta)$ as an operator acting on $L_{\infty}([0, \infty))$ we have $\|\mathscr{A}(\beta)\|_{\infty}=1$.

Definition 2. Given $\beta>0,1<c<2$ and $N \in \mathbf{N}$, define the subspace $\mathscr{H}_{N}^{\frac{1}{N}}(\beta)=$ $\left\{f \in L_{2}(\beta):\left(\varphi_{k}, f\right)_{L_{2}(\beta)}=0, k=0,1, \ldots, N\right\}$.

P4. $\mathscr{H}_{N}^{\frac{1}{N}}(\beta)$ is invariant under $\mathscr{A}(\beta)$.

P5. $\|\mathscr{A}(\beta)\|_{\mathscr{H}_{N}^{\perp}(\beta)}=c^{-(N+1)}$.

Induction hypotheses. Suppose we are given the set of parameters $0<\lambda<1$, $0<D<\infty, N \geqq 3, n_{0} \geqq 0$ and $1<c<2$. At the $n^{\text {th }}$ step we are also given a compact interval $\mathbf{B}^{(n)}=\left[\beta_{-}^{(n)}, \beta_{+}^{(n)}\right] \subset(0, \infty)$. Define the following sets:

$$
\begin{aligned}
& \mathbf{S}_{n}=\left\{x \in \mathbf{A}_{n}: 0 \leqq x \leqq \Delta_{n}^{\frac{1}{3}}\right\}, \\
& \mathbf{D}_{n}=\left\{x \in \mathbf{A}_{n}: \Delta_{n}^{\frac{1}{3}}<x \leqq D \sqrt{n}\right\}, \\
& \mathbf{L}_{n}=\left\{x \in \mathbf{A}_{n}: x>D \sqrt{n}\right\},
\end{aligned}
$$

where $\Delta_{n}=\left(\frac{\sqrt{c}}{2}\right)^{n}$. We state the following induction hypotheses referring to the function $g_{n}(x ; \beta)=\frac{2}{c} Z_{n}^{(q)} p_{n}^{(q)}(x)$ for $\beta \in \mathbf{B}^{(n)}$.

$\mathbf{H}_{1}^{n}$. For each $\beta \in \mathbf{B}^{(n)}$ and $x \in \mathbf{D}_{n}$,

$$
g_{n}(x ; \beta)=L_{n}(\beta) x \exp \left[-\alpha(\beta) x^{2}-\varepsilon_{n}(x ; \beta)\right],
$$

where $\varepsilon_{n}(x ; \beta)$ is given by

$$
\begin{aligned}
\varepsilon_{n}(x ; \beta)= & \left(\frac{2}{c}\right)^{n} B_{1}^{(n)}(\beta) \varphi_{1}(x ; \beta)+\left(\frac{2}{c^{2}}\right)^{n} B_{2}^{(n)}(\beta) \varphi_{2}(x ; \beta) \\
& +\lambda^{n}\left(\frac{2}{c^{2}}\right)^{n} \sum_{j=3}^{N} B_{j}^{(n)}(\beta) \varphi_{j}(x ; \beta) \\
& +Q^{(n)}(x ; \beta)+\frac{1}{x} W^{(n)}(x ; \beta)+R^{(n)}(x ; \beta)
\end{aligned}
$$


The following conditions are met:

(a) $B_{2}^{(n)}(\beta)>0$.

(b) $Q^{(n)}(\cdot ; \beta)$ is defined on $[0, \infty)$ such that $\left|Q^{(n)}(x ; \beta)\right|$, $\left|\frac{\mathrm{d}}{\mathrm{d} x} Q^{(n)}(x ; \beta)\right| \leqq \lambda^{n}\left(\frac{2}{c^{2}}\right)^{n} \forall x \in\left[0, \max \mathbf{D}_{n}+\frac{\Delta_{n}}{2}\right)$.

(c) $W^{(n)}(\cdot ; \beta)$ and $R^{(n)}(\cdot ; \beta)$ are defined on $\mathbf{D}_{n}$ such that $\left|W^{(n)}(x ; \beta)\right| \leqq n^{2} \Delta_{n}$ and $\left|R^{(n)}(x ; \beta)\right| \leqq \lambda^{\frac{3 n}{2}}\left(\frac{2}{c^{2}}\right)^{n}$.

$\mathbf{H}_{2}^{n} \cdot\left|B_{1}^{(n)}(\beta)\right| \leqq \lambda^{n} c^{-n} \forall \beta \in \mathbf{B}^{(n)}$ and $B_{1}^{(n)}\left(\beta_{ \pm}^{(n)}\right)=\mp \lambda^{n} c^{-n}$.

$\mathbf{H}_{3}^{n}$. For each $\beta \in \mathbf{B}^{(n)}$ and $x \in \mathbf{S}_{n}$,

$$
\left|g_{n}(x ; \beta)\right| \leqq 2 \Delta_{n}^{\frac{1}{3}} L_{n}(\beta) \exp \left[-\alpha(\beta) x^{2}\right],
$$

$\mathbf{H}_{4}^{n}$. For each $\beta \in \mathbf{B}^{(n)}$ and $x \in \mathbf{L}_{n}$,

$$
\left|g_{n}(x ; \beta)\right| \leqq L_{n}(\beta) x \exp \left[-\alpha(\beta) x^{2}-\left(\frac{2}{c^{2}}\right)^{n} C^{(n)}(\beta) x^{4}\right],
$$

where $0<C^{(n)}(\beta) \leqq \frac{1}{\sqrt{30}} \gamma(\beta)^{2} B_{2}^{(n)}(\beta)$.

$\mathbf{H}_{5}^{n} . L_{n}, B_{k}^{(n)}(k=1, \ldots, N), C^{(n)}, Q^{(n)}, W^{(n)}$ and $R^{(n)}$ are continuous functions with respect to $\beta$ over the interval $\mathbf{B}^{(n)}$.

Theorem. For each $\sqrt{2}<c<2^{\frac{4}{5}}$ there is a possible choice of the parameters $\lambda, D$, $N$ and $n_{0}$ such that, given the inteval $\mathbf{B}^{\left(n_{0}\right)}$, if the induction hypotheses $\mathbf{H}_{1}^{n_{0}}-\mathbf{H}_{5}^{n_{0}}$ are verified with $Q^{\left(n_{0}\right)}=0$, then for all $n>n_{0}$ there is an interval $\mathbf{B}^{(n)}=\left[\beta_{-}^{(n)}, \beta_{+}^{(n)}\right] \subset \mathbf{B}^{\left(n_{0}\right)}$ for which $\mathbf{H}_{1}^{n}-\mathbf{H}_{5}^{n}$ are verified. The following recursive relations are met:

$\left.\mathbf{R}_{1}^{n}\right) \mathbf{B}^{(n+1)} \subset \mathbf{B}^{(n)}$.

$\left.\mathrm{R}_{2}^{n}\right)$ On $\mathbf{B}^{(n+1)}$ we have $\left\|B_{2}^{(n+1)}-B_{2}^{(n)}\right\|_{\infty} \leqq O\left(\lambda^{\frac{3 n}{2}}\right),\left\|B_{j}^{(n+1)}-\frac{c^{2-j}}{\lambda} B_{j}^{(n)}\right\|_{\infty} \leqq O\left(\lambda^{\frac{n}{2}}\right)$ for $j=3, \ldots, N$ and $C^{(n+1)}(\beta)=C^{(n)}(\beta)-\lambda^{n}$.

$\left.\mathbf{R}_{3}^{n}\right) Q^{(n)}(x ; \beta)=2\left(\mathscr{A}(\beta) Q^{(n)}\right)(x ; \beta)+2 Q_{1}^{(n)}(x ; \beta)$, where $Q_{1}^{(n)}(\cdot ; \beta) \in \mathscr{H}_{N}^{\perp}(\beta) \forall \beta \in \mathbf{B}^{(n)}$, $\left\|Q_{1}^{(n)}(\cdot ; \beta)\right\|_{\infty} \leqq O\left[n^{N} \lambda^{\frac{3 n}{2}}\left(\frac{2}{c^{2}}\right)^{n}\right]$ and $Q_{1}^{(n)}(x ; \beta)=0$ for $x>\max \mathbf{D}_{n+1}+\frac{\Delta_{n+1}}{2}$.

Remark. The condition $Q^{\left(n_{0}\right)}=0$ is not strict, but the statement of the theorem becomes simpler if we adopt it. Note that at the $n_{0}{ }^{\text {th }}$ step all errors may be included in $R^{\left(n_{0}\right)}$.

Remark. From the theorem above, one may conclude that there is a $\beta_{\mathrm{cr}} \in \bigcap_{n=n_{0}}^{\infty} \mathbf{B}^{(n)}$ and a set of initial Hamiltonians (that one may show to be non-void) such that $g_{n}\left(x ; \beta_{\mathrm{cr}}\right)$ (appropriately normalized) converges in probability to const $x \exp \left[-\alpha(\beta) x^{2}\right]$ and therefore, at this critical point, the distribution of $\lim _{n \rightarrow \infty}\left(\frac{c}{4}\right)^{n}\left(\sum_{i \in \Lambda_{n}} \vec{S}_{i}\right)^{2}$ is equal to the corresponding distribution in the classical vector case. 
Corollary 1. The free energy density $f_{n}(\beta)=-2^{-n} \beta^{-1} \ln Z_{n}^{(q)}(\beta)$ admits the following multiscale expansion $\left(n>n_{0}\right)$ :

$$
f_{n}(\beta)=f_{n_{0}}(\beta)-\beta^{-1} \sum_{p=n_{0}+1}^{n} 2^{-p}\left[\ln \left(\frac{2}{c}\right)^{\frac{3}{2}}+O\left[p^{2}\left(\frac{2}{c^{2}}\right)^{p}\right]\right] \forall \beta \in \mathbf{B}^{(n)}
$$

Corollary 2. At the critical temperature one has

$$
\lim _{n \rightarrow \infty} f_{n}\left(\beta_{\mathrm{cr}}\right)=f_{n_{0}}\left(\beta_{\mathrm{cr}}\right)-\beta_{\mathrm{cr}}^{-1} 2^{-n_{0}} \ln \left(\frac{2}{c}\right)^{\frac{3}{2}}+O\left[c^{(-2+\varepsilon) n_{0}}\right] .
$$

\section{Proofs}

Proof of the Theorem. Let us assume inductively that for each $k, n_{0} \leqq k \leqq n$, there is a compact interval $\mathbf{B}^{(k)}$ for which $\mathbf{H}_{1}^{k}-\mathbf{H}_{5}^{k}$ are verified. Furthermore, if $n>n_{0}$ we will assume that $R_{1}^{k}-R_{3}^{k}$ are true for each $k, n_{0} \leqq k \leqq n-1$. Then we will show that there is a compact interval $\mathbf{B}^{(n+1)}$ such that $\mathbf{H}_{1}^{n+1}-\mathbf{H}_{5}^{n+1}$, together with $R_{1}^{n}-R_{3}^{n}$, are verified.

First of all, look at the recurrence relation for $g_{n}(x ; \beta)$, derived from (2.15),

$$
g_{n+1}(x ; \beta)=\exp \left[\beta x\left(x+\Delta_{n+1}\right)\right] \Delta_{n}^{2} \sum_{x_{1}, x_{2} \in \mathbf{A}_{n}}^{\prime \prime} g_{n}\left(x_{1} ; \beta\right) g_{n}\left(x_{2} ; \beta\right)
$$

where the double prime means that the summation is over pairs $\left(x_{1}, x_{2}\right)$ such that $\left|x_{1}-x_{2}\right| \leqq \frac{2}{\sqrt{c}} x \leqq x_{1}+x_{2}$.

For $x \in \mathbf{D}_{n+1}$ we shall divide the sum into three terms (see Fig. 1). Define

$$
\begin{aligned}
& g_{n+1}^{(1)}(x ; \beta)=\exp \left[\beta x\left(x+\Delta_{n+1}\right)\right] \Delta_{n}^{2} \sum_{x_{1}, x_{2} \in \mathbf{D}_{n}}^{\prime \prime} g_{n}\left(x_{1} ; \beta\right) g_{n}\left(x_{2} ; \beta\right), \\
& g_{n+1}^{(2)}(x ; \beta)=\exp \left[\beta x\left(x+\Delta_{n+1}\right)\right] \Delta_{n}^{2} \sum_{\substack{\left\{x_{1} \in \mathbf{S}_{n}, x_{2} \in \mathbf{A}_{n}\right\} \cup \\
\left\{x_{1} \in \mathbf{A}_{n}, x_{2} \in \mathbf{S}_{n}\right\}}}^{\prime \prime} g_{n}\left(x_{1} ; \beta\right) g_{n}\left(x_{2} ; \beta\right), \\
& g_{n+1}^{(3)}(x ; \beta)=\exp \left[\beta x\left(x+\Delta_{n+1}\right)\right] \Delta_{n}^{2} \sum_{\substack{\left\{x_{1} \in \mathbf{L}_{n}, x_{2} \in \mathbf{D}_{n} \cup \mathbf{L}_{n}\right\} \cup \\
\left\{x_{1} \in \mathbf{D}_{n}, x_{2} \in \mathbf{L}_{n}\right\}}}^{\prime \prime} g_{n}\left(x_{1} ; \beta\right) g_{n}\left(x_{2} ; \beta\right) .
\end{aligned}
$$

Assuming that $R_{1}^{k}$ and $R_{2}^{k}$ apply for $n_{0} \leqq k \leqq n-1$, we prove that $\lambda$ may be chosen close enough to 1 so that the $B_{i}^{(n)}$ 's are uniformly bounded by a constant depending only on the values assumed by the $B_{i}^{\left(n_{0}\right)}$ 's on $\mathbf{B}^{\left(n_{0}\right)}$. Then is easy to show that

$$
\left|\varepsilon_{n}(x ; \beta)\right| \leqq O\left[n^{2}\left(\frac{2}{c^{2}}\right)^{n}\right]+O\left[n^{2} \Delta_{n}^{\frac{2}{3}}\right] \quad \forall x \in \mathbb{D}_{n}, \forall \beta \in \mathbf{B}^{(n)}
$$




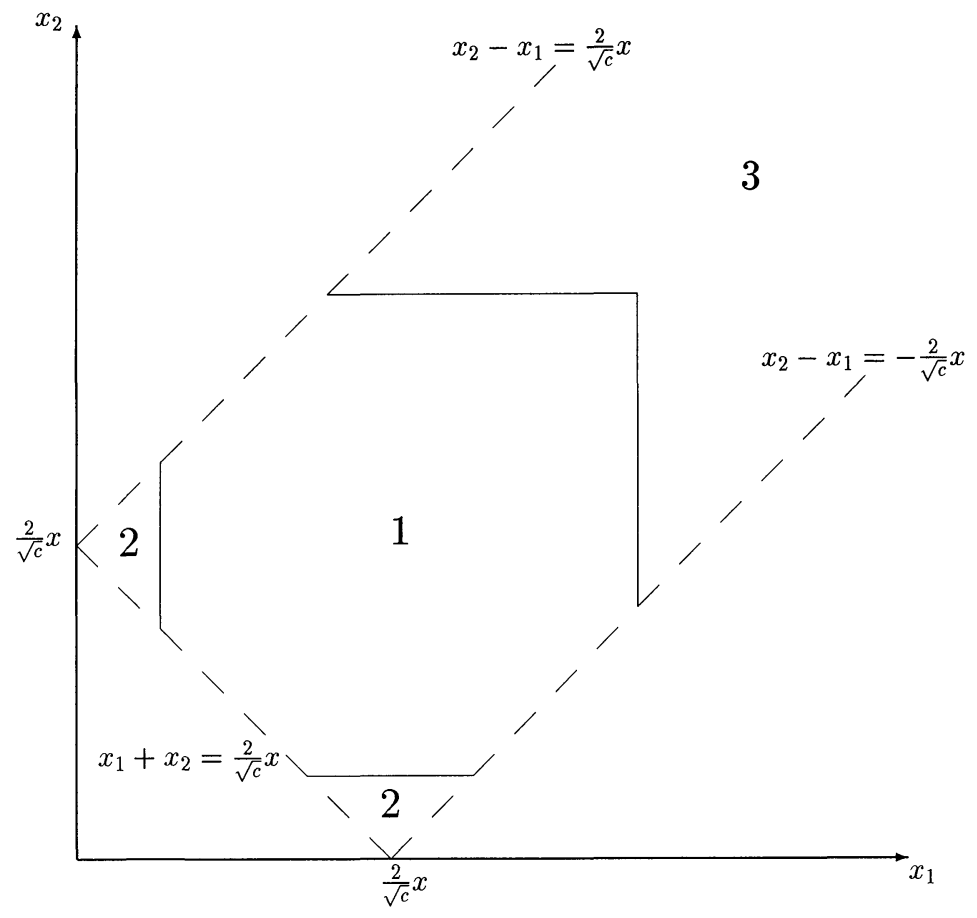

Fig. 1. Regions appearing in $g_{n+1}^{(i)}$

Substituting the form depicted in $\mathbf{H}_{1}^{n}$ and approximating $\exp (-\varepsilon)$ by $1-\varepsilon$ we get

$$
\begin{aligned}
g_{n+1}^{(1)}(x ; \beta)= & L_{n}(\beta)^{2} \exp \left[-\alpha(\beta) x^{2}+\beta \Delta_{n+1} x\right] \Delta_{n}^{2} \sum_{x_{1}, x_{2} \in \mathbf{D}_{n}}^{\prime \prime} x_{1} x_{2}\left[1-2 \varepsilon_{n}\left(x_{1} ; \beta\right)\right. \\
& \left.+T^{(n)}\left(x_{1}, x_{2} ; \beta\right)\right] \exp \left[-\alpha(\beta)\left[x_{1}^{2}+x_{2}^{2}-\frac{2}{c} x^{2}\right)\right]
\end{aligned}
$$

where the truncation error $T^{(n)}$ is clearly $O\left[n^{4}\left(\frac{2}{c^{2}}\right)^{2 n}\right]+O\left(n^{4} \Delta_{n}^{\frac{4}{3}}\right)$.

In order to replace sum by integral we must extend $\varepsilon^{(n)}$ and $T^{(n)}$ over all values of their arguments. In $\varepsilon^{(n)}$ all terms admit natural extensions, except for $W^{(n)}$ and $R^{(n)}$. These two terms, together with $T^{(n)}$, shall be extended as step functions constant over line segments of length $\Delta_{n}$ (or squares of area $\Delta_{n}^{2}$ ) centered in the points of the discrete lattice. Observe that the extensions $\bar{R}^{(n)}, \bar{W}^{(n)}$ and $\bar{T}^{(n)}$ thus obtained preserve their uniform bounds. Now approximate the sum by an integral including an error term $S^{(n)}$ :

$$
\begin{aligned}
& g_{n+1}^{(1)}(x ; \beta) \\
& =L_{n}(\beta)^{2} \exp \left[-\alpha(\beta) x^{2}+\beta \Delta_{n+1} x\left\{\int _ { | x _ { 1 } - x _ { 2 } | \leqq \frac { 2 } { \sqrt { c } } x \leqq x _ { 1 } + x _ { 2 } } x _ { 1 } x _ { 2 } \left[1-2 \bar{\varepsilon}_{n}\left(x_{1} ; \beta\right)\right.\right.\right. \\
& \left.\left.\quad+\bar{T}^{(n)}\left(x_{1}, x_{2} ; \beta\right)\right] \exp \left[-\alpha(\beta)\left(x_{1}^{2}+x_{2}^{2}-\frac{2}{c} x^{2}\right)\right] d x_{1} d x_{2}+S^{(n)}(x ; \beta)\right\} .
\end{aligned}
$$


The symbol $\square$ above means that the integral is taken over the square $x_{1}, x_{2} \in$ $\left[\min \mathbf{D}_{n}-\frac{\Delta_{n}}{2}, \max \mathbf{D}_{n}+\frac{\Delta_{n}}{2}\right]$. The error $S^{(n)}$ may be regarded as the difference between the integral of a function constant over each square of area $\Delta_{n}^{2}$ and the integral of a function coinciding with the former on the centers of the squares located at the points of the discrete lattice. Since we have adopted a convenient extension and have ground bounds over $\frac{\mathrm{d}}{\mathrm{d} x} Q^{(n)}(x ; \beta)$ the error thus obtained is quite small. One may easily check, using standard techniques, that it is inferior to $O\left(\Delta_{n}\right)$. In fact, the dominant contribution to $S^{(n)}$ comes from the border of the integration area, where the profile of the squares does not much the profile of the strip $\left|x_{1}-x_{2}\right| \leqq \frac{2}{\sqrt{c}} x \leqq x_{1}+x_{2}$ (see Fig. 2). This error term is not present in the treatment of the classical scalar model [9], where the domains are linear, and is genuinely $O\left(\Delta_{n}\right)$. Enlarging the area of the borders and integrating we obtained the following estimate:

$$
\left|S^{(n)}(x ; \beta)\right| \leqq O\left(\Delta_{n}\right)\left(1+x^{2}\right) \quad \forall \beta \in \mathbf{B}^{(n)} .
$$

For $x \in \mathbf{D}_{n+1}$ this bound is uniformly majorized by $O\left(n \Delta_{n}\right)$.

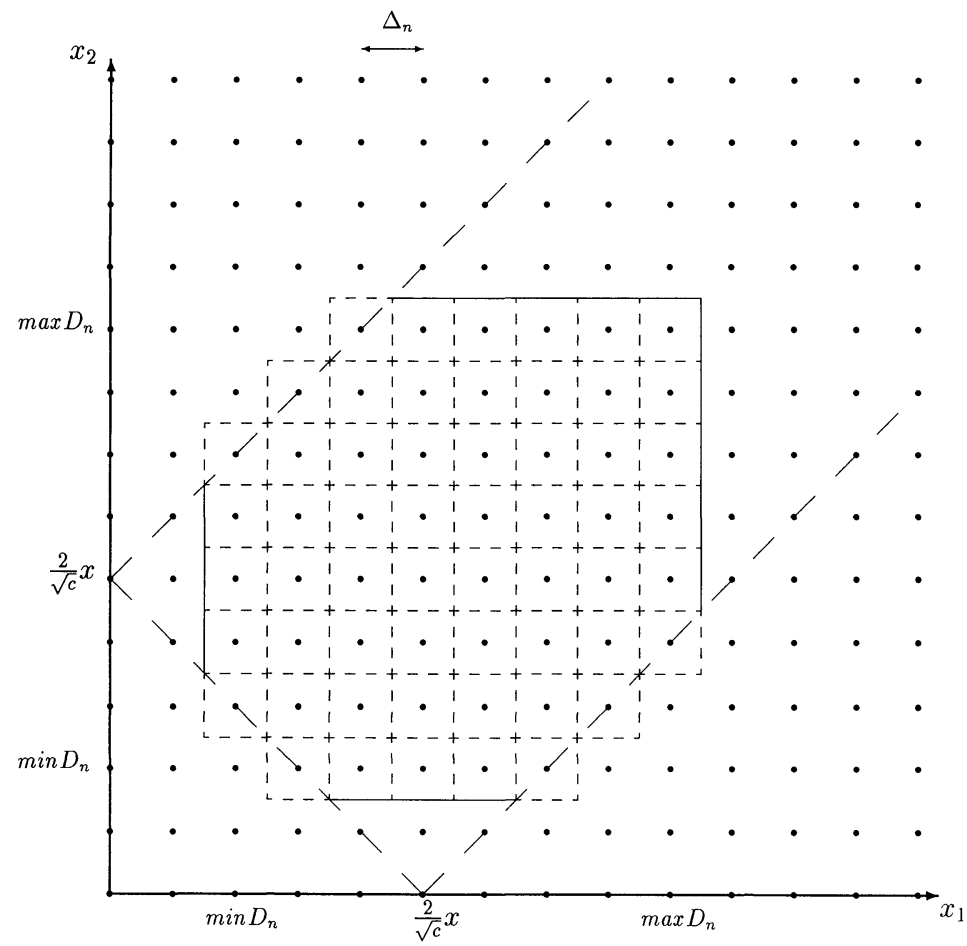

Fig. 2. The approximation of sum by integral in $g_{n+1}^{(1)}$ 
Define the following operator

$$
(\mathscr{B}(\beta) f)(x ; \beta)=\int_{\left|x_{1}-x_{2}\right| \leqq \frac{2}{\sqrt{c}} x \leqq x_{1}+x_{2}} x_{1} x_{2} \exp \left[-\alpha(\beta)\left(x_{1}^{2}+x_{2}^{2}-\frac{2}{c} x^{2}\right)\right] f\left(x_{1} d x_{1} d x_{2} .\right.
$$

We may write

$$
\begin{aligned}
g_{n+1}^{(1)}(x ; \beta)= & L_{n}(\beta)^{2} \exp \left[-\alpha(\beta) x^{2}+\beta \Delta_{n+1} x\right]\left[\mathscr{B}(\beta)\left[1-\bar{\varepsilon}^{(n)}\right]\right)(x ; \beta) \\
& \left.+T_{1}^{(n)}(x ; \beta)+E^{(n)}(x ; \beta)+S^{(n)}(x ; \beta)\right]
\end{aligned}
$$

where

$$
\begin{aligned}
T_{1}^{(n)}(x ; \beta)= & \int_{\left|x_{1}-x_{2}\right| \leqq \frac{2}{\sqrt{c}} x \leqq x_{1}+x_{2}} x_{1} x_{2} \exp \left[-\alpha(\beta)\left(x_{1}^{2}+x_{2}^{2}-\frac{2}{c} x^{2}\right)\right] \\
& \times \bar{T}^{(n)}\left(x_{1}, x_{2} ; \beta\right) d x_{1} d x_{2}
\end{aligned}
$$

is $O\left[n^{4}\left(\frac{2}{c^{2}}\right)^{2 n}\right]+O\left(n^{4} \Delta_{n}^{\frac{4}{3}}\right) \cdot E^{(n)}$ is the error we get when we increase the domain of integration to include the whole strip. The inclusion of the little corners indicated by " 2 " in Fig. 1 adds an error $x O\left(\Delta_{n}\right)$ because the integration area is $O\left(\Delta_{n}^{\frac{2}{3}}\right)$ and the integrand is $x O\left(\Delta_{n}^{\frac{1}{3}}\right)$. The error which arise by the inclusion of region " 3 " may be made arbitrarily small. This happens because $\exp \left[-\alpha(\beta)\left(x_{1}^{2}+x_{2}^{2}-\frac{2}{c} x^{2}\right)\right]$ $\leqq O\left[\exp \left(-D^{2} n\right)\right]$ for $x \in \mathbf{D}_{n+1}$ and $\left(x_{1}, x_{2}\right)$ in "3." Fixing $D$ large enough (but not depending on $n$ ) we may make this error as small as required.

Implementing the change of variables given just after Eq. (2.18) we get an important relation between the operators $\mathscr{B}(\beta)$ and $\mathscr{A}(\beta)$, namely

$$
\mathscr{B}(\beta) f)(x ; \beta)=\sqrt{\frac{\pi}{8 c \alpha(\beta)^{3}}} x(\mathscr{A}(\beta) f)(x ; \beta) \text {. }
$$

Using the explicit diagonalization of $\mathscr{A}(\beta)$ we readily obtain

$$
\begin{aligned}
\left(\mathscr{B}(\beta)\left[1-\bar{\varepsilon}^{(n)}\right]\right)(x ; \beta)= & \sqrt{\frac{\pi}{8 c \alpha(\beta)^{3}}} x\left[1-\left(\frac{2}{c}\right)^{n+1} B_{1}^{(n)}(\beta) \varphi_{1}(x ; \beta)\right. \\
& -\left(\frac{2}{c^{2}}\right)^{n+1} B_{2}^{(n)}(\beta) \varphi_{2}(x ; \beta) \\
& -\lambda^{n+1}\left(\frac{2}{c^{2}}\right)^{n+1} \sum_{j=3}^{N} \frac{c^{2-j}}{\lambda} B_{j}^{(n)}(\beta) \varphi_{j}(x ; \beta) \\
& -2\left(\mathscr{A}(\beta) Q^{(n)}\right)(x ; \beta)-2\left(\mathscr{A}(\beta) \frac{1}{x} \bar{W}^{(n)}\right)(x ; \beta) \\
& \left.-2\left(\mathscr{A}(\beta) \bar{R}^{(n)}\right)(x ; \beta)\right]
\end{aligned}
$$


We write the last term in the form

$$
\left(\mathscr{A}(\beta) \bar{R}^{(n)}\right)(x ; \beta)=\sum_{k=0}^{N} b_{k}^{(n)}(\beta) \varphi_{k}(x ; \beta)+\bar{Q}_{1}^{(n)}(x ; \beta),
$$

where the coefficients $b_{k}^{(n)}(\beta)$ are to be chosen such that

$\int_{0}^{\max \mathbf{D}_{n+1}+\frac{\Delta_{n+1}}{2}} x^{2} \exp \left(-\gamma(\beta) x^{2}\right) \bar{Q}_{1}^{(n)}(x ; \beta) \varphi_{k}(x ; \beta) d x=0 \quad(k=0,1, \ldots, N)$.

Define $\quad Q_{1}^{(n)}(x ; \beta)=\bar{Q}_{1}^{(n)}(x ; \beta) \chi\left[0, \max \mathbf{D}_{n+1}+\frac{\Delta_{n+1}}{2}\right](x)$. It follows that $Q_{1}^{(n)}(\cdot ; \beta) \in \mathscr{H}_{N}^{\frac{1}{N}}(\beta) \in \mathbf{B}^{(n)}$.

The upper bound on $\left|\bar{R}^{(n)}(x ; \beta)\right|$ implies that if $n_{0}$ is sufficiently large, then

$$
\begin{aligned}
&\left|b_{k}^{(n)}(\beta)\right| \leqq O\left[\lambda^{\frac{3 n}{2}}\left(\frac{2}{c^{2}}\right)^{n}\right](k=0,1, \ldots, N) \quad \forall \beta \in \mathbf{B}^{(n)}, \\
&\left\|Q_{1}^{(n)}(\cdot ; \beta)\right\|_{\infty} \leqq O\left[n^{N} \lambda^{\frac{3 n}{2}}\left(\frac{2}{c^{2}}\right)^{n}\right] \quad \forall \beta \in \mathbf{B}^{(n)} .
\end{aligned}
$$

One may easily verify that

$$
\left\|\mathscr{A}(\beta) \frac{1}{x} \bar{W}^{(n)}\right\|_{\infty} \leqq\left\|\bar{W}^{(n)}\right\|_{\infty}\left\|\mathscr{A}(\beta) \frac{1}{x}\right\|_{\infty} \leqq O\left(n^{2} \Delta_{n}\right) .
$$

Adding (4.12) to (4.11) and going back to $g_{n+1}^{(1)}$ we get

$$
\begin{aligned}
g_{n+1}^{(1)}(x ; \beta)= & \sqrt{\frac{\pi}{8 c \alpha(\beta)^{3}}} L_{n}(\beta)^{2} x \exp \left[-\alpha(\beta) x^{2}+\beta \Delta_{n+1} x\right]\left\{1-2 b_{0}^{(n)}(\beta)\right. \\
& -\left(\frac{2}{c}\right)^{n+1}\left[B_{1}^{(n)}(\beta)+c\left(\frac{2}{c}\right)^{-n} b_{1}^{(n)}(\beta)\right] \varphi_{1}(x ; \beta) \\
& -\left(\frac{2}{c^{2}}\right)^{n+1}\left[B_{2}^{(n)}(\beta)+c^{2}\left(\frac{2}{c^{2}}\right)^{-n} b_{2}^{(n)}(\beta)\right] \varphi_{2}(x ; \beta) \\
& -\lambda^{n+1}\left(\frac{2}{c^{2}}\right)^{n+1} \sum_{j=3}^{N}\left[\frac{c^{2-j}}{\lambda} B_{j}^{(n)}(\beta)+\frac{c^{2}}{\lambda} \lambda^{-n}\left(\frac{2}{c^{2}}\right)^{-n} b_{j}^{(n)}(\beta)\right] \varphi_{j}(x ; \beta) \\
& -2\left(\mathscr{A}(\beta) Q^{(n)}\right)(x ; \beta)-2 Q_{1}^{(n)}(x ; \beta)-2\left(\mathscr{A}(\beta) \frac{1}{x} \bar{W}^{(n)}\right)(x ; \beta) \\
& \left.+\sqrt{\frac{8 c \alpha(\beta)^{3}}{\pi}} \frac{1}{x}\left[T_{1}^{(n)}(x ; \beta)+E^{(n)}(x ; \beta)+S^{(n)}(x ; \beta)\right]\right\}
\end{aligned}
$$

Now identify the following terms:

$$
\begin{aligned}
& B_{1}^{(n+1)}(\beta)=B_{1}^{(n)}(\beta)+c\left(\frac{2}{c}\right)^{-n} b_{1}^{(n)}(\beta), \\
& B_{2}^{(n+1)}(\beta)=B_{2}^{(n)}(\beta)+c^{2}\left(\frac{2}{c^{2}}\right)^{-n} b_{2}^{(n)}(\beta),
\end{aligned}
$$




$$
\begin{aligned}
& B_{j}^{(n+1)}(\beta)=\frac{c^{2-j}}{\lambda} B_{j}^{(n)}(\beta)+\frac{c^{2}}{\lambda} \lambda^{-n}\left(\frac{2}{c^{2}}\right)^{-n} b_{j}^{(n)}(\beta)(j=3, \ldots, N), \\
& Q^{(n+1)}(x ; \beta)=2\left(\mathscr{A}(\beta) Q^{(n)}\right)(x ; \beta)+2 Q_{1}^{(n)}(x ; \beta) \\
& W^{(n+1)}(x ; \beta)=-\sqrt{\frac{8 c \alpha(\beta)^{3}}{\pi}} S^{(n)}(x ; \beta) \\
& R_{1}^{(n)}(x ; \beta)=2\left(\mathscr{A}(\beta) \frac{1}{x} \bar{W}^{(n)}\right)(x ; \beta)-\sqrt{\frac{8 c \alpha(\beta)^{3}}{\pi}} \frac{1}{x}\left\{T_{1}^{(n)}(x ; \beta)+E^{(n)}(x ; \beta)\right. \\
&\left.+L_{n}(\beta)^{-2} \mathrm{e}^{\alpha(\beta) x^{2}-\beta \Delta_{n+1} x}\left[g_{n+1}^{(2)}(x ; \beta)+g_{n+1}^{(3)}(x ; \beta)\right]\right\}
\end{aligned}
$$

Thus we may write

$$
\begin{aligned}
g_{n+1}(x ; \beta)= & \sqrt{\frac{\pi}{8 c \alpha(\beta)^{3}}} L_{n}(\beta)^{2} x \exp \left[-\alpha(\beta) x^{2}+\beta \Delta_{n+1} x\right]\left[1-2 b_{0}^{(n)}(\beta)\right. \\
& -\left(\frac{2}{c}\right)^{n+1} B_{1}^{(n+1}(\beta) \varphi_{1}(x ; \beta)-\left(\frac{2}{c^{2}}\right)^{n+1} B_{2}^{(n+1)}(\beta) \varphi_{2}(x ; \beta) \\
& -\lambda^{n+1}\left(\frac{2}{c^{2}}\right)^{n+!} \sum_{j=3}^{N} B_{j}^{(n+1)}(\beta) \varphi_{j}(x ; \beta) \\
& \left.-Q^{n+1}(x ; \beta)-\frac{1}{x} W^{(n+1)}(x ; \beta)-R_{1}^{(n)}(x ; \beta)\right]
\end{aligned}
$$

Approximating $1-\varepsilon$ by $\exp (-\varepsilon)$ and including the corresponding error term $R_{2}^{(n)}$, we get

$$
\begin{aligned}
g_{n+1}(x ; \beta)= & L_{n+1}(\beta) \exp \left[-\alpha(\beta) x^{2}-\left(\frac{2}{c}\right)^{n+1} B_{1}^{(n+1)}(\beta) \varphi_{1}(x ; \beta)\right. \\
& -\left(\frac{2}{c^{2}}\right)^{n+1} B_{2}^{(n+1)}(\beta) \varphi_{2}(x ; \beta) \\
& -\lambda^{n+1}\left(\frac{2}{c^{2}}\right)^{n+1} \sum_{j=3}^{N} B_{j}^{(n+1)}(\beta) \varphi_{j}(x ; \beta)-Q^{(n+1)}(x ; \beta) \\
& \left.-\frac{1}{x} W^{(n+1)}(x ; \beta)-R^{(n+1)}(x ; \beta)\right],
\end{aligned}
$$

where

$$
\begin{aligned}
L_{n+1}(\beta) & =\sqrt{\frac{\pi}{8 c \alpha(\beta)^{3}}} L_{n}(\beta)^{2} \exp \left(-2 b_{0}^{(n)}(\beta)\right), \\
R^{(n+1)}(x ; \beta) & =-\beta \Delta_{n+1}+R_{1}^{(n)}(x ; \beta)-R_{2}^{(n)}(x ; \beta) .
\end{aligned}
$$

So, we have formally reproduced the ansatz in the intermediate fields region, but we still have to show that some estimates are valid. The estimate on $\left|W^{(n+1)}\right|$ derives clearly from the upper bound on $\left|S^{(n)}\right|$. The estimation of $g_{n+1}^{(2)}$ and 
$g_{n+1}^{(3)}$ follows in general terms the same reasoning employed to bound $E^{(n)}$. The outcome is that

$$
\left|g_{n+1}^{(2)}(x ; \beta)\right| \leqq L_{n}(\beta)^{2} O\left(\Delta_{n}\right) x \exp \left(-\alpha(\beta) x^{2}\right) \quad \forall x \in \mathbf{D}_{n+1}, \forall \beta \in \mathbf{B}^{(n)},
$$

and if $D$ is fixed large enough

$$
\left|g_{n+1}^{(3)}(x ; \beta)\right| \leqq L_{n}(\beta)^{2} O\left(\Delta_{n}^{\frac{4}{3}}\right) \exp \left(-\alpha(\beta) x^{2}\right) \quad \forall \beta \in \mathbf{D}_{n+1}, \forall \beta \in \mathbf{B}^{(n)} .
$$

Using the estimates so far obtained, one may bound $\left|R_{1}^{(n)}\right|$ as follows:

$$
\begin{aligned}
\left|R_{1}^{(n)}(x ; \beta)\right| & \leqq O\left(n^{2} \Delta_{n}\right)+\frac{1}{x}\left\{O\left[n^{4}\left(\frac{2}{c^{2}}\right)^{2 n}\right]+O\left(n^{4} \Delta_{n}^{\frac{4}{3}}\right)\right\} \\
& \leqq O\left[n^{4} \Delta_{n}^{-\frac{1}{3}}\left(\frac{2}{c^{2}}\right)^{2 n}\right]+O\left(n^{4} \Delta_{n}\right) \quad \forall x \in \mathbf{D}_{n+1}, \forall \beta \in \mathbf{B}^{(n)}
\end{aligned}
$$

In the interval $\sqrt{2}<c<2^{\frac{4}{5}}$ we may choose $\lambda$ depending on $c$ sufficiently close to 1 such that if $n_{0}$ is sufficiently large, then

$$
\left|-\beta \Delta_{n+1} x+R_{1}^{(n)}(x ; \beta)\right| \leqq \lambda^{2 n}\left(\frac{2}{c^{2}}\right)^{n} \quad \forall x \in \mathbf{D}_{n+1}, \forall \beta \in \mathbf{B}^{(n)} .
$$

Note that $R_{3}^{n}$ applies from the very definition of $Q^{(n+1)}$. Iterating $R_{3}^{k}$, with $n_{0} \leqq k \leqq n$, we get

$$
Q^{(n+1)}(x ; \beta)=\sum_{k=n_{0}}^{n} 2^{n+1-k}\left(\mathscr{A}(\beta)^{n-k}\left(Q_{1}^{(k)}\right)(x ; \beta) .\right.
$$

Observe that we are using $Q^{\left(n_{0}\right)}=0$. Take $\omega \in\left[\frac{n_{0}}{n_{0}+1}, 1\right)$ and define $n_{1}=[n \omega]$. In order to estimate the sum above up to the term $k=n_{1}$ we shall need the following lemma of Tauberian type:

Lemma. Given $\beta \in\left[\beta_{-}, \beta_{+}\right] \subset(0, \infty)$ and $f \in \mathscr{H}_{N}^{\perp}(\beta),\|f\|_{\infty} \leqq 1$, there are constants $N_{0}, M$ and $L$ depending on $c, \beta_{-}$and $\beta_{+}$such that, if $N \geqq N_{0}$ and $p \geqq 1$, then

L1. $\left|\left(\mathscr{A}(\beta)^{p} f\right)(x ; \beta)\right| \leqq \exp \left(-\frac{2 \ln c}{9} p N\right) \quad \forall x \leqq M \sqrt{p N}$.

L2. $\left|\frac{d}{d x}\left(\mathscr{A}(\beta)^{p} f\right)(x ; \beta)\right| \leqq 2 L^{\frac{p}{2}} \exp \left(-\frac{\ln c}{9} p N\right) \quad \forall x \leqq \frac{1}{2} M \sqrt{p N}$.

The proof of this lemma will be left to the end. First of all, note that if $k \leqq n_{1}$, then $n-k \geqq n(1-\omega)$. So it is possible to choose $N$ depending on $\omega, c, \beta_{-}^{\left(n_{0}\right)}, \beta_{+}^{\left(n_{0}\right)}$ and $D$ so large that $\max \mathbf{D}_{n+1}+\frac{\Delta_{n+1}}{2} \leqq \frac{1}{2} M\left(c, \beta_{-}^{n_{0}}, \beta_{+}^{\left(n_{0}\right)}\right) \sqrt{(n-k) N}$ for all $k \leqq n_{1}$. Then for $x \leqq \max \mathbf{D}_{n+1}+\frac{\Delta_{n+1}}{2}$ the lemma tells us that

$$
\begin{aligned}
& \left|\sum_{k=n_{0}}^{n_{1}} 2^{n+1-k}\left(\mathscr{A}(\beta)^{n-k} Q_{1}^{(k)}\right)(x ; \beta)\right| \\
& \quad \leqq \sum_{k=n_{0}}^{n_{1}} 2^{n+1-k} \exp \left[-\frac{2 \ln c}{9}(n-k) N\right] O\left[k^{N} \lambda^{\frac{3 k}{2}}\left(\frac{2}{c^{2}}\right)^{k}\right] .
\end{aligned}
$$


Choosing $N$ so large that $2 \exp \left(-\frac{2 \ln c}{9} N\right) \leqq\left(\lambda^{2} 2 c^{-2}\right)^{\frac{1}{1-\omega}}$ we get

$$
\left[2 \exp \left(-\frac{2 \ln c}{9} N\right)\right]^{n-k} \leqq \lambda^{2 n}\left(\frac{2}{c^{2}}\right)^{n}
$$

and the sum up to order $k=n_{1}$ turns out to be less or equal to $O\left[\lambda^{2 n}\left(\frac{2}{c^{2}}\right)^{n}\right]$.

To estimate the remaining terms of the sum, use $\mathbf{P 3}$ and the uniform bounds on $Q_{1}^{(k)}$;

$$
\begin{aligned}
\left|\sum_{k=n_{1}+1}^{n} 2^{n+1-k}\left(\mathscr{A}(\beta)^{n-k} Q_{1}^{(k)}\right)(x ; \beta)\right| & \leqq \sum_{k=n_{1}+1}^{n} 2^{n+1-k} O\left[k^{N} \lambda^{\frac{3 k}{2}}\left(\frac{2}{c^{2}}\right)^{k}\right] \\
& \leqq \text { const } 2^{n(1-\omega)} n^{N+1}\left(\lambda^{\frac{3}{2}} \frac{2}{c^{2}}\right)^{n \omega}
\end{aligned}
$$

Choosing $\omega$ so close to 1 that $2^{1-\omega}\left(\lambda^{\frac{3}{2}} 2 c^{-2}\right)^{\omega} \leqq x^{\frac{5}{4}} 2 c^{-2}$ we see that the second term is of order $O\left[n^{N+1} \lambda^{\frac{5 n}{4}}\left(\frac{2}{c^{2}}\right)^{n}\right]$, yielding the upper bound on $\left|Q^{(n+1)}(x ; \beta)\right|$ required in $\mathbf{H}_{1}^{n+1}$.

To verify the bound on $\left|\frac{\mathrm{d}}{\mathrm{d} x} Q^{(n+1)}(x ; \beta)\right|$, we follow basically the same steps. We estimate the corresponding sum up to order $k=n_{1}$ using the second part of the lemma and the remainder terms using the easily derived property

$$
\left\|(\mathscr{A}(\beta) f)^{\prime}\right\|_{\infty} \leqq \operatorname{const}(\beta)\|f\|_{\infty},
$$

together with the uniform bounds on $Q_{1}^{(k)}$. The only term that should be treated separately is $2 \frac{\mathrm{d}}{\mathrm{d} x} Q_{1}^{(n)}(x ; \beta)$, but again from $\mathbf{P 3}$ we easily get that this term is less than or equal to $O\left[n^{N-\frac{1}{2}} \lambda^{\frac{3 n}{2}}\left(\frac{2}{c^{2}}\right)^{n}\right]$ for $x<\max \mathbf{D}_{n+1}+\frac{\Delta_{n+1}}{2}$ and $\beta \in \mathbf{B}^{(n)}$.

Inserting all the estimates obtained so far, one may verify that $\lambda$ may be chosen in $(0,1)$ such that

$$
\left|R_{2}^{(n)}(x ; \beta)\right| \leqq \lambda^{2 n}\left(\frac{2}{c^{2}}\right)^{n} \quad \forall x \in \mathbf{D}_{n+1}, \forall \beta \in \mathbf{B}^{(n)} .
$$

Estimates (4.24) and (4.30) prove the required upper bound on $\left|R^{(n+1)}\right|$.

To finish the verification of $\mathbf{H}_{1}^{(n+1)}$ note that

$$
\begin{aligned}
B_{2}^{(n+1)}(\beta)= & B_{2}^{\left(n_{0}\right)}(\beta)+\sum_{k=n_{0}}^{n-1}\left(B_{2}^{(k+1)}(\beta)-B_{2}^{(k)}(\beta)\right) \\
& >\inf \left\{B_{2}^{\left(n_{0}\right)}(\beta): \beta \in \mathbf{B}^{\left(n_{0}\right)}\right\}-\sum_{k=n_{0}}^{n-1} O\left(\lambda^{\frac{3 k}{2}}\right) \\
& >\frac{1}{2} \inf \left\{B_{2}^{\left(n_{0}\right)}(\beta): \beta \in \mathbf{B}^{\left(n_{0}\right)}\right\}>0 \quad \forall \beta \in \mathbf{B}^{(n)}
\end{aligned}
$$

provided that $n_{0}$ is sufficiently large. 
We now consider the question of determining the interval $\mathbf{B}^{(n+1)}$. From estimate (4.14) we see that $B_{2}^{n+1}\left(\beta_{-}^{(n)}\right)>\lambda^{n+1} c^{-(n+1)}$ and $B_{1}^{n+1}\left(\beta_{+}^{(n)}\right)<-\lambda^{n+1} c^{-(n+1)}$. The continuity of $B_{1}^{(n+1)}$ then implies that there are two numbers, $\beta_{-}^{(n+1)}$ and $\beta_{+}^{(n+1)}$, such that $\beta_{-}^{(n)}<\beta_{-}^{(n+1)}<\beta_{+}^{(n+1)}<\beta_{+}^{(n)}$ and $\mathbf{H}_{2}^{n+1}$ is satisfied with the definition $\mathbf{B}^{(n+1)}=\left[\beta_{-}^{(n+1)}, \beta_{+}^{(n+1)}\right]$. Repeating this process, one generates a sequence of nested compact intervals. In the end, $\beta_{\mathrm{cr}}$ will lie in the intersection of all $\mathbf{B}^{(n)}$ 's. This is the usual Bleher-Sinai prescription for finding the critical temperature.

Let us now check $\mathbf{H}_{3}^{n+1}$. Here we follow an approach similar to the one employed to verify $\mathbf{H}_{1}^{n+1}$, with the simplification of working only with upper bounds. It is enough to note that for $y \in \mathbf{D}_{n} \cup \mathbf{L}_{n}$ we may adopt the estimate

$$
\left|g_{n}(y ; \beta)\right| \leqq \frac{6}{5} L_{n}(\beta) y \exp \left[-\alpha(\beta) y^{2}\right] .
$$

Substitution in the recurrence relation (4.1) yields

$\left|g_{n+1}(x ; \beta)\right| \leqq L_{n}(\beta)^{2} \exp \left[-\alpha(\beta) x^{2}+\beta \Delta_{n+1} x\right]\left[\frac{36}{25}(\mathscr{B}(\beta) 1)(x ; \beta)+V^{(n)}(x ; \beta)\right]$

where $V^{(n)}$ stands for all error terms. Using the same techniques already introduced, it is straightforward to check that $\left|V^{(n)}(x ; \beta)\right| \leqq O\left(\Delta_{n}\right)$ for $x \in \mathbf{S}_{n+1}$. From the definitions of $\mathscr{B}(\beta)$ and $L_{n+1}(\beta)$ it follows that

$$
\left|g_{n+1}(x ; \beta)\right| \leqq L_{n+1}(\beta) \exp \left[-\alpha(\beta) x^{2}+\beta \Delta_{n+1} x+2 b_{0}^{(n)}(\beta)\right]\left[\frac{36}{25} x+O\left(\Delta_{n}\right)\right],
$$

$\mathbf{H}_{3}^{n+1}$ then follows from the fact that $x \leqq \Delta_{n}{ }_{n+1}^{\frac{1}{3}}$ in $\mathbf{S}_{n+1}$ and from (4.14).

The proof of $\mathbf{H}_{4}^{n+1}$ presents some new features. To begin with, note that if $x \in \mathbf{L}_{n+1}$ then condition $x_{1}+x_{2} \geqq \frac{2}{\sqrt{c}} x$ implies that $x_{1}$ and $x_{2}$ cannot be simultaneously smaller than $\frac{D \sqrt{n}}{2 \sqrt{c}}$. In fact, we could drop the " 2 " in the denominator, but for technical reasons we shall keep it. Divide $g_{n+1}$ into two terms, namely

$$
\begin{aligned}
& g_{n+1}^{(A)}(x ; \beta)=\exp \left[\beta x\left(x+\Delta_{n+1}\right)\right] \Delta_{n}^{2} \sum_{x_{1}, x_{2} \geqq \frac{D \sqrt{n}}{2 \sqrt{c}}}^{\prime \prime} g_{n}\left(x_{1} ; \beta\right) g_{n}\left(x_{2} ; \beta\right), \\
& g_{n+1}^{(B)}(x ; \beta)=2 \exp \left[\beta x\left(x+\Delta_{n+1}\right)\right] \Delta_{n}^{2} \sum_{x_{1} \geqq \frac{D \sqrt{n}}{2 \sqrt{c}}, x_{2}<\frac{D \sqrt{n}}{2 \sqrt{c}}}^{\prime \prime} g_{n}\left(x_{1} ; \beta\right) g_{n}\left(x_{2} ; \beta\right) .
\end{aligned}
$$

For $x \sim \sqrt{n}$ the leading term in $\varepsilon^{(n)}$ is clearly $\left(\frac{2}{c^{2}}\right)^{n} B_{2}^{(n)}(\beta) \sqrt{\frac{2}{15}} \gamma(\beta)^{2} x^{4}$. The positivity of this term is guaranteed by inequality (4.31) (with $n+1$ replaced by $n$ ). 
So it is easy to prove that for $x \in \mathbf{D}_{n}, x \geqq \frac{D \sqrt{n}}{2 \sqrt{c}}$, the following estimate applies:

$$
\varepsilon_{n}(x ; \beta)>\frac{1}{2} \sqrt{\frac{2}{15}} \gamma(\beta)^{2}\left(\frac{2}{c^{2}}\right)^{n} B_{2}^{(n)}(\beta) x^{4}>\left(\frac{2}{c^{2}}\right)^{n} C^{(n)}(\beta) x^{4} .
$$

This result means that the large fields upper bound (3.5) may be used for all $x \geqq \frac{D \sqrt{n}}{2 \sqrt{c}}$. To estimate $g_{n+1}^{(A)}$ insert this bound and use that $x_{1}+x_{2} \geqq \frac{2}{\sqrt{c}} x$ implies $x_{1}^{4}+x_{2}^{4} \geqq \frac{2}{c^{2}} x^{4}$. Then

$$
\begin{aligned}
\left|g_{n+1}^{(A)}(x ; \beta)\right| \leqq & L_{n}(\beta)^{2} \exp \left[-\alpha(\beta) x^{2}-\left(\frac{2}{c^{2}}\right)^{n+1} C^{(n)}(\beta) x^{4}+\beta \Delta_{n+1} x\right] \\
& \times \Delta_{n}^{2} \sum_{x_{1}, x_{2} \geqq \frac{D \sqrt{n}}{2 \sqrt{c}}}^{\prime \prime} x_{1} x_{2} \exp \left[-\alpha(\beta)\left(x_{1}^{2}+x_{2}^{2}-\frac{2}{c} x^{2}\right)\right] \\
= & L_{n}(\beta)^{2} \exp \left[-\alpha(\beta) x^{2}-\left(\frac{2}{c^{2}}\right)^{n+1} C^{(n)}(\beta) x^{4}+\beta \Delta_{n+1} x\right] \\
& \times\left[\int_{\left|x_{1}-x_{2}\right| \leqq \frac{2}{\sqrt{c}} x \leqq x_{1}+x_{2}} x_{1} x_{2}\left[-\alpha(\beta)\left(x_{1}^{2}+x_{2}^{2}-\frac{2}{c} x^{2}\right)\right] d x_{1} d x_{2}\right. \\
& \left.+U^{(n)}(x ; \beta)\right] .
\end{aligned}
$$

where the symbol $*$ stands for the restriction of the domain of integration to a set of squares compatible with the sum. Since the integrand is positive, we may majorize this integral by dropping the restriction and thus obtaining $(\mathscr{B}(\beta) 1)(x ; \beta)$. The error relative to the approximation of sum by integral may be estimated analogously to the estimation of $S^{(n)}$. One verifies that

$$
\left|U^{(n)}(x ; \beta)\right| \leqq O\left(\Delta_{n}\right) x^{2} \quad \forall x \in \mathbf{L}_{n+1}, \forall \beta \in \mathbf{B}^{(n+1)}
$$

Using the properties of $\mathscr{B}(\beta)$, the definition of $L_{n+1}(\beta)$ and taking into account that $c<2^{\frac{4}{5}}$, we see that $\lambda$ may be chosen to that

$$
\left|g_{n+1}^{(A)}(x ; \beta)\right| \leqq L_{n+1}(\beta) x \exp \left[-\alpha(\beta) x^{2}-\left(\frac{2}{c^{2}}\right)^{n+1}\left(C^{(n)}(\beta)-\frac{1}{2} \lambda^{n}\right) x^{4}\right] \text {. }
$$

Concerning $g_{n+1}^{(B)}$, one may check that if $D$ is chosen large enough $\left|g_{n+1}^{(B)}(x ; \beta)\right| \leqq L_{n+1}(\beta) x \exp \left[-\alpha(\beta) x^{2}-\left(\frac{2}{c^{2}}\right)^{n+1} C^{(n)}(\beta) x^{4}\right] O\left[\lambda^{n}\left(\frac{2}{c^{2}}\right)^{n}\right]$.

So, it follows that, for $x \in \mathbf{L}_{n+1}$ and $\beta \in \mathbf{B}^{(n+1)}$,

$$
\left|g_{n+1}(x ; \beta)\right| \leqq L_{n+1}(\beta) x \exp \left[-\alpha(\beta) x^{2}-\left(\frac{2}{c^{2}}\right)^{n+1}\left(C^{(n)}(\beta)-\lambda^{n}\right) x^{4}\right] \text {. }
$$


We satisfy $\mathbf{H}_{4}^{n+1}$ defining $C^{(n+1)}(\beta)=C^{(n)}(\beta)-\lambda^{n}$. The required upper and lower bounds on $C^{(n+1)}$ are easily verified, as well as the continuity statements in $\mathrm{H}_{5}^{n+1}$.

Proof of the lemma. We follow closely [9]. First of all, note that there is a constant $L\left(c, \beta_{+}\right)$such that

$$
\left\|\frac{\mathrm{d}^{m}}{\mathrm{~d} x^{m}}(\mathscr{A}(\beta) f)\right\|_{\infty} \leqq L\left(c, \beta_{+}\right)\|f\|_{\infty}(m=0,1,2) \quad \forall \beta \in\left[\beta_{-}, \beta_{+}\right] .
$$

Let us suppose that there is a $x_{0} \leqq M \sqrt{p N}$ (the value of $M$ will be fixed later) such that

$$
\left(\mathscr{A}(\beta)^{p} f\right)\left(x_{0} ; \beta\right)>\exp \left(-\frac{2 \ln c}{9} p N\right) .
$$

The case with $\left(\mathscr{A}(\beta)^{p} f\right)\left(x_{0} ; \beta\right)$ negative may be treated analogously.

Define $\Gamma=L^{-p} \exp \left(-\frac{2 \ln c}{9} p N\right)$, fix $\beta \in\left[\beta_{-}, \beta_{+}\right]$and construct the function

$$
h(x)= \begin{cases}\left(\mathscr{A}(\beta)^{p} f\right)\left(x_{0} ; \beta\right)-L^{p}\left(x-x_{0}\right. & \text { if } x_{0} \leqq x \leqq x_{0}+\Gamma \\ 0 & \text { otherwise }\end{cases}
$$

Clearly $h(x)^{2} \leqq\left(\mathscr{A}(\beta)^{p} f\right)(x ; \beta)^{2} \forall x \in[0, \infty)$.

It is easy to check that $\|f\|_{\mathscr{K}_{N}^{1}(\beta)} \leqq\|f\|_{\infty} \leqq 1$. Together with property P5, this implies that

$$
\begin{aligned}
c^{-p(N+1)} & \geqq \sqrt{\frac{4}{\sqrt{\pi}} \gamma(\beta)^{\frac{3}{2}} \int_{0}^{\infty} x^{2} \exp \left(-\gamma(\beta) x^{2}\right)\left(\mathscr{A}(\beta)^{p} f\right)(x ; \beta)^{2} d x} \\
& \geqq \sqrt{\frac{4}{\sqrt{\pi}} \gamma(\beta)^{\frac{3}{2}} \int_{x_{0}}^{x_{0}+\Gamma} x^{2} \exp \left(-\gamma(\beta) x^{2}\right)\left[\left(\mathscr{A}(\beta)^{p} f\right)\left(x_{0} ; \beta\right)-L^{p}\left(x-x_{0}\right)\right]^{2} d x} \\
& \geqq \operatorname{const}\left(c, \beta_{-}, \beta_{+}\right) L^{p} \Gamma^{\frac{5}{2}} \exp \left[-\gamma(\beta)(M \sqrt{p N}+\Gamma)^{2}\right] \\
& =\operatorname{const}\left(c, \beta_{-}, \beta_{+}\right) \Gamma^{\frac{-3 p}{2}} \exp \left[-\gamma(\beta)(M \sqrt{p N}+\Gamma)^{2}-\frac{5 \ln c}{9} p N\right] .
\end{aligned}
$$

Hence

$$
-p(N+1) \ln c \geqq-\gamma(\beta)(M \sqrt{p N}+\Gamma)^{2}-\frac{5 \ln c}{9} p N-\frac{3}{2} p \ln L+\text { const. }
$$

Rearranging terms we obtain the inequality

$$
\begin{aligned}
0 \geqq & {\left[\frac{4}{9} \ln c-\gamma(\beta) M^{2}\right] p N-2 \gamma(\beta) M \Gamma \sqrt{p N} } \\
& +\left[p\left(\ln c-\frac{3}{2} \ln L\right)-\gamma(\beta) \Gamma^{2}+\text { const }\right] .
\end{aligned}
$$


Define $M=\sqrt{\ln c / 3 \gamma\left(\beta_{+}\right)}$to obtain, for all $p \geqq 1$,

$$
0 \geqq \frac{1}{9} \ln c N+\text { const } \sqrt{N}+\text { const }^{\prime} .
$$

Since $c>1$, there is a $N_{0}\left(c, \beta_{-}, \beta_{+}\right)$such that the left-hand side of (4.48) is positive for any $N \geqq N_{0}$. The contradiction proves $\mathbf{L 1}$.

In order to show $\mathbf{L 2}$, assume that there is a $x_{0} \leqq \frac{1}{2} M \sqrt{p N}$ such that

$$
\left(\mathscr{A}(\beta)^{p} f\right)^{\prime}\left(x_{0} ; \beta\right)>2 L^{\frac{p}{2}} \exp \left(-\frac{\ln c}{9} p N\right) .
$$

From (4.43) one may conclude that $\left\|\left(\mathscr{A}(\beta)^{p} f\right)^{\prime \prime}\right\|_{\infty} \leqq L^{p}\|f\|_{\infty}$, implying that $\left(\mathscr{A}(\beta)^{p} f\right)^{\prime}(x ; \beta) \geqq\left(\mathscr{A}(\beta)^{p} f\right)^{\prime}\left(x_{0} ; \beta\right)-L^{p}\left(x-x_{0}\right)$ for all $x \geqq x_{0}$. So

$$
\left(\mathscr{A}(\beta)^{p} f\right)(x ; \beta) \geqq\left(\mathscr{A}(\beta)^{p} f\right)\left(x_{0} ; \beta\right)+\left(x-x_{0}\right)\left(\mathscr{A}(\beta)^{p} f\right)^{\prime}\left(x_{0} ; \beta\right)-\frac{L^{p}}{2}\left(x-x_{0}\right)^{2} .
$$

Then, from $\mathbf{L} 1$ and (4.49), it follows that

$$
\left(\mathscr{A}(\beta)^{p} f\right)(x ; \beta)>-\exp \left(-\frac{2 \ln c}{9} p N\right)+2\left(x-x_{0}\right) L^{\frac{p}{2}} \exp \left(-\frac{\ln c}{9} p N\right)-\frac{L^{p}}{2}\left(x-x_{0}\right)
$$

Let $\tilde{x}=x_{0}+2 L^{-\frac{p}{2}} \exp \left(-\frac{\ln c}{9} p N\right)$. We see from (4.51) that

$$
\left(\mathscr{A}(\beta)^{p} f\right)(\tilde{x} ; \beta)>\exp \left(-\frac{2 \ln c}{9} p N\right) .
$$

But clearly $\tilde{x} \leqq M \sqrt{p N}$ for $N$ big enough. The contradiction of L1 proves L2. This completes the proof of the lemma and also the proof of our main theorem.

Proof of the Corollaries. Use that $Z_{n}^{(q)}=\frac{c}{4} \Delta_{n} \sum_{x \in \mathbf{A}_{n}}\left(2 x+\Delta_{n}\right) g_{n}(x ; \beta)$ to obtain

$$
\begin{aligned}
f_{n+1}(\beta)-f_{n}(\beta)= & -\beta^{-1} 2^{-(n+1)} \ln \left\{\frac{4}{c} \frac{L_{n+1}(\beta)}{L_{n}(\beta)^{2}}\right. \\
& \left.\times \frac{\Delta_{n+1} \sum_{x \in A_{n+1}}\left(2 x+\Delta_{n+1}\right) L_{n+1}(\beta)^{-1} g_{n+1}(x ; \beta)}{\left[\Delta_{n} \sum_{x \in \mathrm{A}_{n}}\left(2 x+\Delta_{n}\right) L_{n}(\beta)^{-1} g_{n}(x ; \beta)\right]^{2}}\right\} .
\end{aligned}
$$

From the induction hypotheses one may verify that

$$
\Delta_{n} \sum_{x \in \mathbf{A}_{n}}\left(2 x+\Delta_{n}\right) L_{n}(\beta)^{-1} g_{n}(x ; \beta)=\frac{1}{2} \sqrt{\frac{\pi}{\alpha(\beta)^{3}}}+O\left[n^{2}\left(\frac{2}{c^{2}}\right)^{n}\right] \quad \forall \beta \in \mathbf{B}^{(n)} \text {. }
$$

Using (4.18g) and (4.14) one gets

$$
f_{n+1}(\beta)-f_{n}(\beta)=-\beta^{-1} 2^{-(n+1)}\left[\ln \left(\frac{2}{c}\right)^{\frac{3}{2}}+O\left[n^{2}\left(\frac{2}{c^{2}}\right)^{n}\right]\right]
$$

Iterating (4.55) one easily proves the Corollaries. 
Acknowledgements. This work was partially supported by Conselho Nacional de Desenvolvimento Científico e Tecnológico (CNPq), Brazil.

\section{References}

1. Bleher, P.M., Major, P.: Renormalization of Dyson's hierarchical vector valued $\phi^{4}$ model at low temperatures. Commun. Math. Phys. 95, 487-532 (1984)

2. Bleher, P.M., Major, P.: The large-scale limit of Dyson's hierarchical vector-valued model at low temperatures. The non-Gaussian case. I: Limit theorem for the average spin II: Description of the large scale limit. Ann. Inst. Henri Poincaré, Phys. Théor. 49, No. 1, 7-85, 87-143 (1988).

3. Bleher, P.M., Sinai, Ya.G.: Investigation of the critical point in models of the type of Dyson's hierarchical models. Commun. Math. Phys. 33, 23-42 (1973)

4. Dyson, F.: Existence of a phase transition in a one-dimensional Ising ferromagnet. Commun. Math. Phys. 12, 91-107 (1969)

5. Gawedzki, K., Kupiainen, A.: Triviality of $\phi_{4}^{4}$ and all that in a hierarchical model approximation. J. Stat. Phys. 29, 683-698 (1982)

6. Gawedzki, K., Kupiainen, A.: Massless lattice $\phi_{4}^{4}$ theory: Rigorous control of a renormalizable asymptotically free model. Commun. Math. Phys. 99, 197-252 (1985)

7. Schor, R., O'Carroll, M.: Low Temperature Properties of the Hierarchical Classical Vector Model. Commun. Math. Phys. 138, 487-505 (1991)

8. Sewell, G.L.: Quantum theory of collective phenomena. Oxford: Clarendon Press, 1986

9. Sinai, Ya.G.: Theory of phase transitions: Rigorous results. London: Pergamon Press, 1982

Communicated by M.E. Fisher 